CRITICA, Revista Hispanoamericana de Filosofia

Vol. XXII, No. 66 (diciembre 1990): 75-85

\title{
A CRUCIAL DISTINCTION: INITIAL DATA AND LAW APPLICATION INSTANCES *
}

EDUARDo H. FlichmaN

Universidad de Buenos Aires

SADAF

\section{Introduction}

When we reflect about natural science theories, a typical problem often emerges with regard to distinguishing the diversity of roles played by the statements of a theory. Some of them form what many philosophers like to call "the hard core" of the theory. Others are related to pressuposed or to auxiliary theories. Others, finally, state consequences which are almost always dependent on auxiliary statements which are not-strictly - part of the theory; but which are complements to it, necesary to obtaining interesting consequences regarding testing. They are what are currently called "data", "initial data", "initial conditions", "boundary conditions", "concrete circumstances", etc.

I would like to remark that, often, I will not explicitly distinguish between reference to statements of a theory and reference

- This paper was read at the X Simposio de Filosoffa (Instituto de Investigaciones Filosóficas, Universidad Nacional Autónoma de México), held in Mexico City in August 27th-29th, 1990. It was also read as a conference in SADAF (Sociedad Argentina de Análisis Filosófico), Buenos Aires, Argentina, in October 16th, 1990. I am grateful to the authorities of the Instituto de Investigaciones Filosoficas, UNAM, which made possible my participation in the Symposium, to Alberto Moretti and Raúl Orayen, for their very helpful comments on earlier drafts of this paper, and to the other participants on both meetings, for the illuminating discussions on this and related items. I am grateful too to Guillermo Pissinis for checking my English. 
to the matter those statements are about. For instance, when the expression "initial data" appears, it can refer to a statement of initial data or to the corresponding circumstances to which the statement refers. The contex will have the tacit task, in each case, of indicating the correct reference, except in those cases where an explicit indication is required for the sake of clarity. In such cases, as usual, I will employ double quotation marks in order to indicate that the referent is a linguistic expression.

The expressions "initial conditions" and "boundary conditions" have been taken from physics. The second one is almost never used in philosophical literature (at least in natural science philosophy): it becomes substituted by any of the other expressions mentioned above. The first one, on the other hand, is used with a much more extensive meaning that in physics. For instance, the expression "initial" lose, in the extension, its essentially temporal content. It retains a logical sense: it is a (minor) premiss in a deduction.

For example, if we conjoin with the premiss "All ravens are black", the (minor) premiss "Charlie is a raven" we can deduce the conclusion "Charlie is black". Natural science philosophers would usually call the premiss "Charlie is a raven" an "initial condition". This practice is very far from the usage of physicists with respect to the same expression, even if the physicist's use is included in the more extensive one of philosophers. For instance, if we add to Newton's laws of particle dynamics and of universal gravitation, statements which indicate position and velocity at some determinate moment of all the particles involved in a (practically) isolated system - and whose particles are subject only to gravitational interaction among them-and if the system continues to be isolated from that moment on, during a certain period of time, physicists will call that moment an "initial instant"; and they will call those positions and velocities, "initial position" and "initial velocity", respectively. Moreover, they will speak of the fact that the system remains isolated during the indicated period as a "boundary 
condition", and the fact that "such and such" a particle has "such and such" initial position and initial velocity as an "initial condition". (In fact, this example of the usage of "boundary condition" is not strictly correct; but it is, perhaps, a good intuitive approach, in order to avoid technical details.)

Initial and boundary conditions (more that those mentioned above) will allow us to solve the Newtonian equations with a single result. In our special example, the problem consists in obtaining the position of each particle of the system during the indicated period. This solution tells us, in particular, the position and velocity of each particle in the final instant of that period. It is a deduced conclusion from initial and boundary conditions (minor premisses) and from the Newtonian equations (mayor premisses). In this example we can see that the physicist's use of "initial conditions" is a special case of the philosopher's use, which is much more extensive. Philosophers often use the expressions "datum", "initial datum" and "concrete circumstance" as synonymous with "initial condition". This does not mean that they always accept such synonymy; but they currently do so.

I think that the enlarged use of those expressions by natural science philosophers, eliminating any previously established discrimination and without introducing new discriminations, may have been one of the sources of the great difficulties in clarifying the problem of the epistemological demarcation between accidental uniformities and natural laws.

The purpose of this paper is to open the way to begin an approach to the solution of this vexing and recalcitrant problem.

\section{The role of initial data and the role of application instances} in natural science theories

It is my purpose to support the idea that, within the structure of theories, and in the articulation among theories, there must be carefully distinguished those auxiliary statements which play 
the role of initial data from those playing a very different role, that of application instances, which are generally confused with the first.

The distinction is not related with the process of theory construction but rather with the structure of theories whose fundamental statements have been previously delineated, at least on first approach.

The basic idea is the following: the (main and auxiliary) roles of the statements of theories are:

A. The role of GENERAL LAWS of the structure. They are universal and not localized statements (without proper names or definite descriptions). If the theory is axiomatized, they are the (interpreted) AXIOMS of the theory.

$B$. The role of CONCRETE CIRCUMSTANCES. They are localized statements (with at least one proper name or definite description) and not universal.

$C$. The role of APPLICATION INSTANCES. They are localized and, generally, singular statements. Main role: They instanciate general and derivative laws (see role and definition $D$ ) localizing them and thus producing law instances (see role and definition $E$ ). They can also instanciate law instances (if the last ones are universally quantified). Complementary role: They can also instanciate data (see role $F$ and definition $G$ ) when these dat $a$ are universally quantified.

$D$. The role of THEOREMS I, proved at the last step, from (and only from) general laws (role $A$ ). general laws are excluded from role $D$ because they play role A. THEOREMS I ane called "DERIVATIVE LAWS".

$E$. The role of THEOREMS II, proved, at the last step, from (and only from) general laws and application instances. Theorems II are called "LAW INSTANCES".

$F$. The role of THEOREMS III, proved, at the last step, from (and 
only from) general laws, application instances (which may be absent) and concrete circumstances.

$G$. I will call ("INITIAL" or "FINAL") "DATA", the statements which play roles $B$ and $F$. Each final datum may be, in its turn, an initial datum (premiss) in the proof of another theorem III. Role $B$ corresponds only to initial data, while role $F$ may correspond to initial or to final data.

3. What are the respective roles of natural laws and accidental uniformites?

$H$. My proposal: NATURAL LAWS play, in the structure of theories, the roles $A$ or $D$ (general or derivative laws).

I. My proposal: ACCIDENTAL UNIFORMITIES play, in the structure of theories, the role $F$ (data which are theorems III, when those data are universally quantified).

$J$. Finally, I will call "COSMIC ACCIDENTS" the accidental uniformities without localization and with an indefinite-and perhaps infinite-number of universally quantified particulars.

\section{The application instances}

Natural laws always possess at least one universal quantifier: "In all instants, all pieces of metal modify their linear dimensions in such and such way when their temperature is modified". "In all instants, on each particle there is some total force acting upon it such that particle is accelerated in such and such way". Etc.

Let us take the first example. I must first remark that the over simplification in the statement of the law left tacit the fact that, in the 'such and such way in which the linear dimensions of the piece of metal are modified' is included the null modification, when the modification of the temperature is null; that is, when the piece of metal is neither heated nor cooled. The same argument applies to the other example. 
I will now conjoin to the law of the first example (which, depending on the theoretical frame in which it is involved, may be a general or a derivative law). Taken as the major premiss, the following minor premiss: "the beginning of the twelfth stroke of the bell in the tower of the Buenos Aires Townhall during the night from July the 5th to July the 6th, 1990 is an instant". I will abbreviate with " $A$ " the expression: "the beginning of the twelfth stroke of the bell in the tower of the Buenos Aires Townhall during the night from July the 5th to July the 6th, 1990". From both premisses we can deduce: "At $A$, all the pieces of metal to which the temperature is modified, modify their linear dimensions in such and such a way".

The conclusion of the deductive argument is a law instance, which has been localized (instanciated) in that particular instant. Of course, the minor premiss has not been an initial datum (in spite of its temporality) but an application instance. IN ALL CASES WHERE A DEDUCTION ELIMINATES A UNIVERSAL QUANTIFIER OF A GENERAL OR DERIVATIVE LAW (OR FROM A LAW INSTANCE) BY MEANS OF THE CONJUNCTION WITH A (GENERALLY) SINGULAR LOCALIZED PREMISS, SUCH PREMISS IS AN APPLICATION INSTANCE.

Let us notice that we may repeat the operation with the other universal quantifier of our law. We conjoin to the law instance previously obtained, the minor premiss: "The horseshoe which Peter, the blacksmith, put on the back right leg of my horse is a piece of metal". I will abbreviate "the horseshoe which Peter, the blacksmith, put on the back right leg of my horse" with " $B$ ". I will now suppose, only for the sake of simplicity of the example, that the blacksmith was doing his work on my horse just after $A$. I will also suppose that he had heated the horseshoe in order to give to it the correct shape, just at instant $A$. Then, if we now conjoin this singular premiss to the law instance, we obtain the conclusion: "At $A, B$, whose temperature was modified, changed its linear dimensions in such and such a way". 
We obtain once again a law instance, in this case localized to our particular instant and to our particular piece of metal. The second singular premiss conjoined is again an application instance, because its conjunction with the law instance allowed the elimination of another universal quantifier form that law instance.

Let us look now into the second example, the first-and-second principle of Newton: "For each instant and for each particle, either the total force on the particle in that instant is not null, and in that case it is proportional to its acceleration and the constant of proportionality depends only on the particle (not on time) and it is furthermore a positive number; or the total force on it in that instant is null and its acceleration is also null."

We can see that here we must also recognize the possibility of null total force with null acceleration (principle of inertia or the first principle of Newton), which corresponds to the case of null modification of the temperature and null change in linear dimensions, in the first example.

Therefore, if we instanciate time and particle in the example of dynamics, conjoining premisses: " $A$ is an instant" and "The mite of dust which stands on my finger is a particle", we obtain the following conclusion: "Either the total force acting upon the mite of dust which stands on my finger at $A$, is not null, and in that case the value of that force equals the product of the value of the acceleration and a number, and such number is positive; or the total force on that mite at that moment is null and its acceleration is also null." We can see that the conclusion is a law instance of the same law of Newton, localized to that instant and to that particle. Both singular premisses, conjoined with the law, allowed the deduction of a law instance where the universal quantifiers were eliminated. Therefore, those minor premisses are application instances. 


\section{The initial data}

We will now study another example, in this case an accidental uniformity, with role $F$ (theorem III), an accidental uniformity at last with respect to the framework of a certain theory.

The statement is: "All ravens are black". We return to Kneale's famous example, but completely modified and seen from a different point of view. This example is interesting because the number of individuals involved is indefinite and it could even be infinite (for instance, in an eternal universe, where there are always ravens from some instant forth). On the other hand, there is no localization. These features produce more confusion with respect to the decision regarding its being a natural law or an accidental uniformity. The indetermination of number and the absence of localization, make it a typical example of a cosmic accident, in the case that it turns out to be an accidental uniformity.

We will consider the case (the only important case regarding our problem) in which the feature of 'being black' is not one of the definitory features of ravens.

In a zoological taxonomic classification according to more or less conventional and prescientific systems (we may suppose that it is stated in XVIIth century), this statement is isolated. It is not articulated in a theory. Therefore, in that case it has no sense (from our point of view) to inquire whether it is a natural law or an accidental uniformity. Let us suppose that we succeed in finding (in a later time) a theory or a bundle of theories from which our statement can be deduced as a final datum if we conjoin the theories with initial data. This result will mean that it is an accidental uniformity; more particulary, a cosmic accident. Let us suppose again, that we have the synthetic theory of evolution (mutation, selection of the fittest, etc.) and that we have also an explication of "raven" in the context of that theory, which does not sensibly modify its definitory features, so that our statement remains valid within its frame (because what we 
call "ravens" in this theory are approximately the same animals as before).

that it is possible to deduce "All ravens are piack Irom" inis theory and from initial data such as: 1 . The climate and other environmental conditions in the region of the Earth where the predecessor of ravens were originated were such and such, at a time previous to $t_{0}$. 2 . Certain mutations which were produced in these animals during a period $T$, beginning at $t_{0}$, gave occasion for the generation of a new species, the ravens. 3. Such mutations produced black ravens. 4. Other mutations of the same animals, in the same period $T$ (which would have produced white ravens) did not come out, not even as eggs. 5. The climate and other environmental conditions in that region on Earth during the period $T$ was such and such.

We may suppose that those initial data conjoined with the synthetic theory of evolution, allow us to deduce that the potential mutant white ravens could not be formed due to genetic problems related to environmental conditions, while the mutant black ravens developed without problems (because of their good adaptability to the condictions ruling in that region and time). If we now conjoin new initial data about conditions in periods later than $T$, which are also final data taken from meteorological, physical, chemical, geological and echological theories, it will be possible to derive "All ravens are black".

Thus, we see that "All ravens are black" plays the role of final datum WITHIN THE FRAME OF THAT THEORY. In particular, it is an accidental uniformity (because it is universally quantified) and, specifically, a cosmic accident.

This example show us that the initial data necessary to derive our statement, have not been used in order to eliminate universal quantifiers from the laws or instances of laws of the theory involved. This result clearly shows that these data are not application instances. 
We may note that we can also use application instances in order to eliminate the universal quantifier in "All ravens are black", even if our statement is not a natural law or a law instance (role $C$ of application instances allows it). We may conjoin the (minor) premiss "Charlies is a raven" (application instance) to our statement, and deduce the conclusion "Charlie is black". Such a conclusion is also a final datum.

\section{Conclusion}

We may conclude that a correct distinction between application instances and initial data allows us to attempt to discriminate between natural laws and accidental uniformities, always in relation to a certain theoretical framework. It may also allow us to find that distinction by studying the roles played by those statements in theories, within a non-essentialist line; that is, without involving the concept of natural necessity and, on the other hand, without having to apply counterfactual conditionals, which carried and carry so many problems.

This proposal may also allow us to show how one and the same statement may be a natural law when it is articulated in the structure of a theory (or bundle of theories) and an accidental uniformity when it is integrated in the structure of another theory (or bundle of theories). This, in its turn, allows us to show that it is impossible to distinguish natural laws from accidental uniformities by means of logical form. But I will not treat of those issues here.

Recibido: 2 actubre 1990. 


\section{RESUMEN}

Dos tipos de enunciados auxiliares que integran $\longrightarrow$ si bien no estrictamente- la estructura de las teorías de las ciencias naturales, se suelen confundir entre sí: nos referimos a los datos iniciales y a las instancias de aplicación. Un análisis profundo de sus diferencias permite la posibilidad de distinguir, en un segundo paso, las leyes naturales de las uniformidades accidentales en teorías ya constituidas. Los datos iniciales agregados a leyes generales o derivadas permiten deducir un dato final que, si posee cuantificación universal se puede identificar con una uniformidad accidental. En cambio, las instancias de aplicación, agregadas a leyes generales o derivadas, permiten la deducción de instancias de ley, eliminando cuantificadores universales. 